\title{
Monitoring texture formation during diamond growth by specular and diffuse reflectance interferometry
}

Article in Diamond and Related Materials · September 2016

DOI: 10.1016/j.diamond.2016.08.013

CITATIONS

0
READS

19

5 authors, including:

\section{Colin Delfaure}

Atomic Energy and Alternative Energies Com. .

11 PUBLICATIONS 20 CITATIONS

SEE PROFILE

\section{Pascal Ponard}

Thales Electron Devices

16 PUBLICATIONS 32 CITATIONS

SEE PROFILE
Jean-Paul Mazellier

Thales Group

29 PUBLICATIONS 148 CITATIONS

SEE PROFILE

\section{Samuel Saada}

Atomic Energy and Alternative Energies Com...

71 PUBLICATIONS 568 CITATIONS

SEE PROFILE

Some of the authors of this publication are also working on these related projects: 


\title{
Monitoring texture formation during diamond growth by specular and diffuse reflectance interferometry
}

\author{
Colin Delfaure ${ }^{\mathrm{a}, *}$, Nicolas Tranchant $^{\mathrm{a}}$, Jean-Paul Mazellier ${ }^{\mathrm{b}}$, Pascal Ponard ${ }^{\mathrm{c}}$, Samuel Saada $^{\mathrm{a}}$ \\ ${ }^{a}$ CEA, LIST, Diamond Sensors Laboratory, Gif-sur-Yvette Cedex 91191, France \\ ${ }^{\mathrm{b}}$ Thales Research and Technology, Route Dèpartementale 128, Palaiseau 91767, France \\ ${ }^{\mathrm{c}}$ Thales Electron Devices, ZI de Vongy, Thonon-les-Bains 74202, France
}

\section{A R T I C L E I N F O}

\section{Article history:}

Received 27 June 2016

Received in revised form 27 August 2016

Accepted 27 August 2016

Available online 5 September 2016

\section{Keywords:}

Diamond growth

Texture

Sequential synthesis

LRI

Scattered light

\begin{abstract}
A B S T R A C T
This paper is aimed at describing a sequential synthesis coupled to an extensive analysis in order to study the morphological evolution of a $\{100\}\{111\}\langle 100\rangle$ textured diamond film. We compare several morphological parameters obtained by in situ optical methods and ex situ characterizations. The texture formation is found to be correlated with a significant increase in the growth rate of the film. In addition, morphology reconstruction of polycrystalline film is presented from which the $R_{100}$ covering ratio of $\{100\}$ faces is extracted. As a result, a phenomenological model has been developed for real-time growth parameters extraction of the film based on in situ optical measurements.
\end{abstract}

(c) 2016 Elsevier B.V. All rights reserved.

\section{Introduction}

Diamond film morphologies can be controlled during growth through a careful tuning of process parameters: temperature, pressure, gas composition and microwave power. The $\{100\}\{111\}\langle 100\rangle$ diamond film morphology (notation from Ref. [1]) is often reported under peculiar conditions and is due to a prevailing texture $[2,3]$, associated to competitive process described by Van der Drift [4]. It leads to polycrystalline films with relatively smooth surface, i.e. Root Mean Square (RMS) roughness below $100 \mathrm{~nm}$, and is of great interest for applications needing diamond with a controlled roughness such as X-ray windows, low-friction coatings, photonic crystals [5-8]. This approach is very complementary to Chemical Mechanical Polishing (CMP) or other smoothing processes because it reduces polishing step or even suppress it depending on the application requirements [9-11]. This paper aims at providing a new optical method to monitor diamond films morphology during its synthesis.

The Laser Reflectance Interferometry (LRI) method is often used to follow etch/growth rate and optical constants of different materials [12-16]. It is particularly well-suited for diamond synthesis because of the low absorption of diamond in visible range [6,17-21].

\footnotetext{
* Corresponding author at: CEA, LIST, Centre CEA de Saclay, Bâtiment 451 PC45,
} Gif-Sur-Yvette 91191, France.
We report use of a combined LRI set-up in which specular and scattered intensities are collected. A step-by-step synthesis coupled with a methodological analysis of the texture formation in terms of morphology, covering ratio, selection rate, roughness and growth rate is presented. Ex situ measurement of growth rate, roughness and morphology were performed after each sequential step and results were correlated to LRI signal intensities. A statistical model for crystal selection and texture formation is proposed. It is used to extract the growth parameters and selection rates of two similar synthesis by fitting growth rate evolution versus time. This model is relevant for real-time monitoring of texture during the synthesis.

\section{Sequential growth}

\subsection{Experimental details}

Substrates are $10 \times 10 \mathrm{~mm}^{2}$ cleaved from $400 \mu \mathrm{m}$ thick silicon wafers with $\langle 100\rangle$ orientation. An initial step of nanoseeding is necessary to initiate diamond growth on the surface [22]. In order to follow the $\{100\}\{111\}\langle 100\rangle$ texture evolution, thick films need to be grown (at least several micrometers). Thus, process parameters have been optimized not only for $\{100\}\{111\}\langle 100\rangle$ texture formation but also to achieve a relatively high growth rate (of at least $1 \mu \mathrm{m} / \mathrm{h}$ ). The reactor is an homemade Microwave Plasma Chemical Vapor Deposition (MPCVD) reactor with $2.45 \mathrm{GHz}$ microwave generator. 
Optimized growth parameters are a pressure of 90 mbar, $1.5 \mathrm{~kW}$ total power, a gas composition of 4 vol.\% of $\mathrm{CH}_{4}, 1 \mathrm{vol} . \%$ of $\mathrm{O}_{2}$, $300 \mathrm{ppm}$ of $\mathrm{N}_{2}$ and $95 \mathrm{vol} . \%$ of $\mathrm{H}_{2}$ for a total flow of $240 \mathrm{sccm}$. Substrate temperature is $900{ }^{\circ} \mathrm{C}$ as measured with a pyrometer $(1.45-1.80 \mu \mathrm{m})$. A sequential step-by-step synthesis, divided into 10 successive stages, is performed. First stage refers to the nanoseeding, second stage is a fixation stage which last 5 min only during which the growth is initiated from the nanodiamonds. Other growth stage durations are $2000 \mathrm{~s}$. Different ex situ analyses are performed on the sample (as described below) which is thereafter reintroduced in the reactor for the following growth stage. Every single stage starts with $5 \mathrm{~min}$ of $\mathrm{H}_{2}$ plasma to ensure that the following growth step starts at a reproducible temperature of $900^{\circ} \mathrm{C}$. Between each step, the sample is weighted to calculate the growth rate. Its roughness is measured with an Atomic Force Microscope (AFM) in contact mode on a $100 \mu \mathrm{m}^{2}$ area. The surface morphology is observed with a Carl Zeiss Supra40 High Resolution Scanning Electron Microscope (HRSEM). In that case a specific technique of zone marking is used to observe the exact same sample area after successive growth steps. Three different marked areas are studied with this technique to provide a representative measurement over the surface. An image analysis treatment on HRSEM pictures using ImageJ software [23], provides the surface covering ratio $R_{100}$ of the marked area at each step.

An optical set-up is developed combining a classical LRI set-up and an additional photodiode to measure the surface scattered light at grazing angles. As presented on Fig. 1, $1 \mathrm{~mW}, 670 \mathrm{~nm}$ linearlypolarized laser diode is shined at $45^{\circ}$ on the surface of the growing diamond film and two photodiodes collect intensities of both scattered $\left(I_{s c}\right)$ and specular $\left(I_{s p}\right)$ lights. To subtract thermal background induced noise as well as plasma light, the beam impinging on the sample surface is chopped at $18 \mathrm{~Hz}$ and the diodes are connected to lock-in amplifiers used to extract $I_{s c}$ and $I_{s p}$ with a signal to noise ratio better than 4000 . As the scattered light is considered to fall on a Lambertian surface, meaning that the scattered intensity is constant regardless of the angle of view [24], only a small fraction of this intensity falls on the corresponding photodiode. The system sensitivity loss is then compensated by using a laser line filter at $670 \mathrm{~nm}$ in front of the detection diode. Both intensities are recorded together with synthesis parameters during growth via a computer interface. Both reflectances $R_{s c}$ and $R_{s p}$ are calculated by taking the ratio of diode measured intensity to initial intensity. The latter is measured by recording the signal reflected by a bare substrate prior to any deposition step.

Simple reflectance models exist for MPCVD and Molecular Beam Epitaxy (MBE) processes to extract optical constants $n$ and $k$, roughness $\delta$ and growth rate $G$ from $R_{s p}[18,19,21,25]$. As this signal results from interferences in the diamond layer, $G$ is computed from the

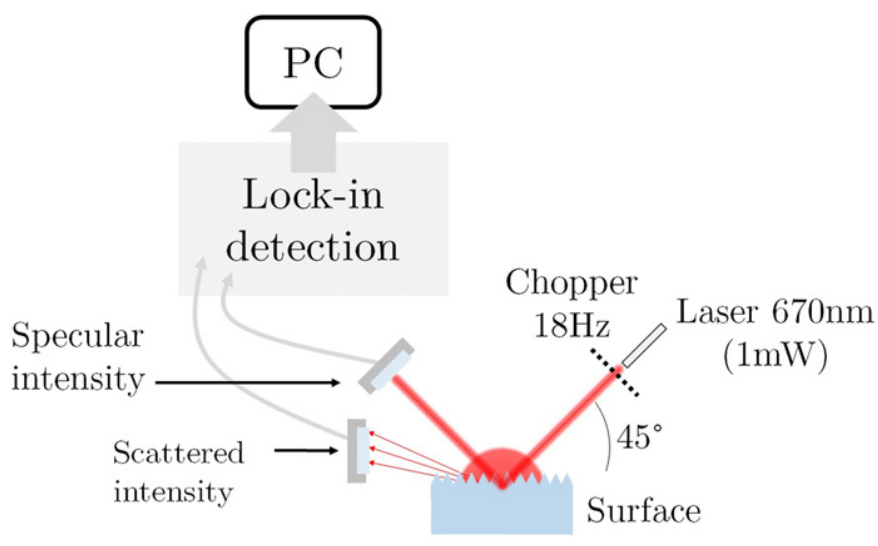

Fig. 1. Sketch of combined LRI setup used during growth of diamond films. signal oscillation period. Roughness is calculated from the oscillation amplitude changes and from global reflectivity. However, when roughness increases, oscillations amplitude decreases until the signal becomes too weak to be measured. As non-textured polycrystalline diamond roughness scales up with film thickness, LRI technique can only be used for thin layers $(<5 \mu \mathrm{m})$. Indeed scattered light offers here a complementary informations to follow growth even for thick films.

\subsection{Results}

\subsubsection{Surface covering ratio}

HRSEM pictures presented on Fig. 2 are taken from the exact same zone of the film surface after each of the ten growth steps. Four faces are colorized to follow more easily the competitive growth. There is a clear indication that the number of $\{100\}$ faces emerging on the surface in the first hour of growth is less than the initial nanoseeding density. Stabilization of the roughness is clearly visible and is correlated to texture evolution.

Covering ratio $R_{100}$ is defined as the ratio of $\{100\}$ surface area to the total area analyzed (about $100 \mu \mathrm{m}^{2}$ ). This parameter can be used in numerical computation of diamond texture process [26]. Image treatment via ImageJ routines were performed to extract $R_{100}$. Three representative areas of the surface have been observed to increase the statistical accuracy of this method. The mean evolution of $R_{100}$ on the three areas is plotted on Fig. 3 and shows a stabilization after $3 \mathrm{~h}$. The ratio does not reach $100 \%$ as the twist between crystals prevent two neighbors with a similar orientation but different twist angles to form a completely smooth surface.

\subsubsection{Roughness and growth rate}

Specular reflectivity $\left(R_{s p}\right)$ measured during this step-by-step synthesis is presented on Fig. 4. Uncertainty on sample positioning between each step has a detrimental effect on intensities overlapping but trends can still be interpreted. Two regimes can be observed from LRI signal. First, amplitude is quickly decaying as growing grains increase the roughness. Then oscillations can be seen again as $\{100\}$ faces start to form a smooth surface after $3 \mathrm{~h}$ in this particular case.

Still on Fig. 4 is presented the scattered reflectivity $\left(R_{s c}\right)$ which is increasing and stabilizing after $3 \mathrm{~h}$. A similar trend to covering ratio is observed. The oscillations already observed on $R_{s p}$ can be seen on $R_{s c}$ and correspond to the interference phenomena between reflected light from the rough surface and reflected light reflected which has traveled twice within the film thickness [17]. As theoretically expected, there is almost an half-wavelength phase shift between specular interferences and scattered interferences due to the Fresnel radiation phase shift respectively at diamond/gas and diamond/substrate interfaces [27].

By using the model of Ref. [25], the surface roughness can be extracted from the oscillations amplitude. This model corresponds to multilayer scalar scattering due to surface roughness and is based on a Gaussian definition of the surface height distribution. It is presented on Fig. 5 with experimental data measured between each step. Roughness is first increasing due to growth of grains. As $\{100\}$ faces are joining, the roughness is slowly decreasing. This trend is obviously driving $R_{s p}$ behavior. Difference between the AFM results and LRI data lies in the roughness definition of each method.

The growth rate is also extracted from $R_{s p}$ by measuring oscillations period over growth time. These data presented on Fig. 6 are compared to experimental growth rate measured by differential weighing between each steps. Error bars are calculated by taking into account the uncertainty of sample surface determination which is measured on a photography of the sample. Despite the data dispersion there is a clear increase in the growth rate confirmed by ex situ measurements. The growth rate is almost doubled within 


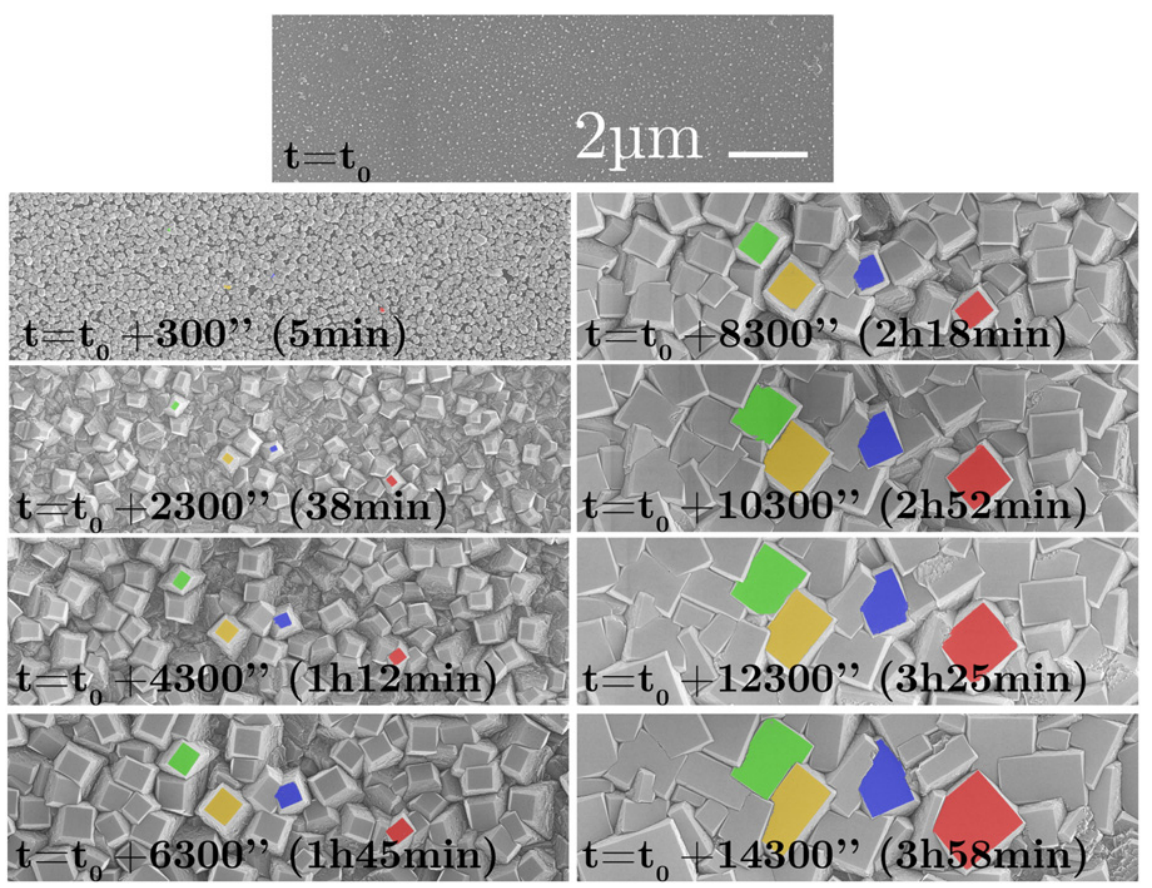

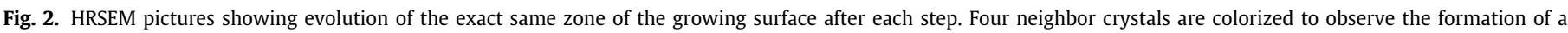
smooth surface.

$4 \mathrm{~h}$. Although the first oscillations are usually used in the literature to determine the growth rate $[20,28,29]$, this result demonstrate that the effective growth rate is strongly evolving when this texture is forming.

The influence of nitrogen on the growth rate of various faces of diamond crystals has been extensively studied [30-33]. The texture formation and the induced increase in growth rate $G$ is here attributed to the nitrogen in the gas phase. As suggested for $\{100\}\{111\}\langle 100\rangle$ textured diamond films [25], this growth rate increase results from the progressive burial of the slowest growing $\{111\}$ faces.

From this step-by-step experiment, we observe in one hand that texture formation induces a growth rate increase and on the other hand that roughness is increasing at first and slowing down as crystals are joining. Roughness evolution is related to the completeness

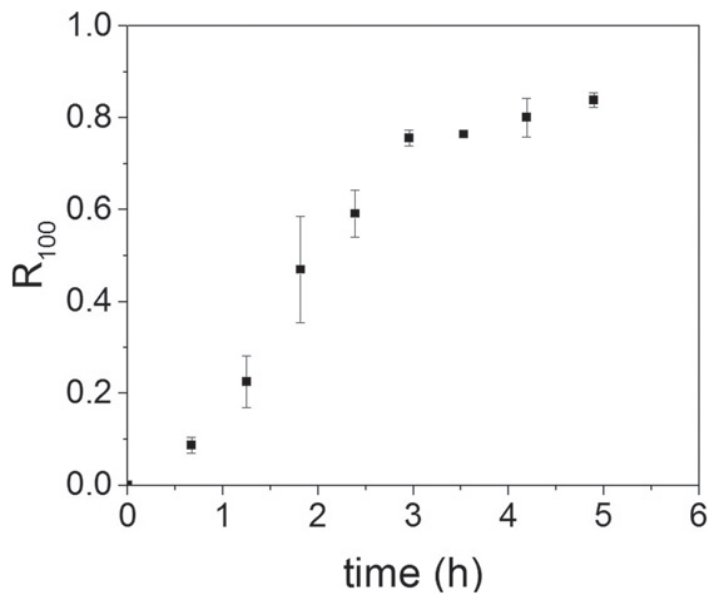

Fig. 3. Covering ratio $R_{100}$ measured via image analysis of several HRSEM pictures of the same zone. Measurement is reproduced on three different areas and the mean value is represented with standard deviation as error bars. of texture hence to the $\{100\}$ faces area ratio. This ratio is noted $R_{100}$ and is zero at the very beginning of growth. It tends to reach unity as crystal selection proceed. When looking at $R_{s c}$ signal one can assume there is a direct correlation between the optical signal and the covering ratio $R_{100}$. A simple, yet effective model is developed in the following to derive this correlation from optical signals.

\section{Texture modeling}

Numerical simulations are often used to model 2D, 3D synthesis of diamond films that grow according to Van der Drift competitive process [16,26,34-39]. A simpler approach is used here for texture modeling. To account for different morphologies observed during diamond growth, the parameter $\alpha=\sqrt{3} \cdot v_{100} / v_{111}$ is used [7]. This "growth parameter" is often used in the diamond literature to describe the morphology of crystals. It is not a direct controllable

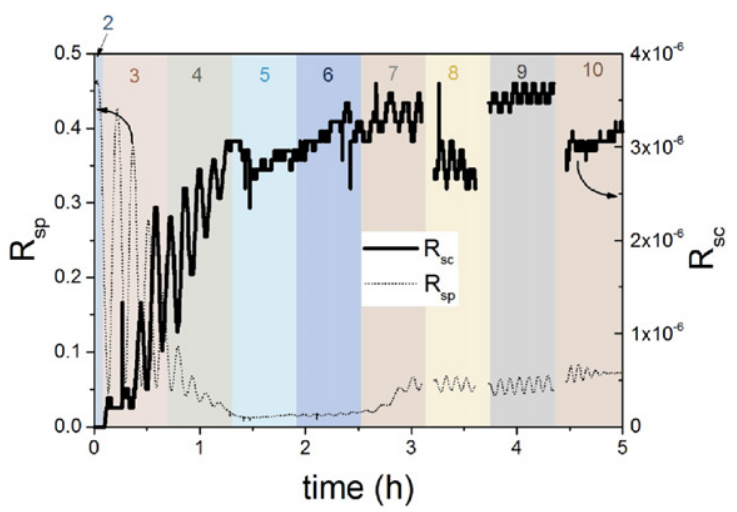

Fig. 4. Signals recorded during sequential step-by-step synthesis, divided into 10 successive stages (first step corresponds to nanoseeding, second step is for fixation purpose, following steps correspond to film growth). Specular reflectance $R_{s p}$ (thin doted line) is represented on left scale. Scattered reflectance $R_{s c}$ (thick solid line) is represented on right scale. 


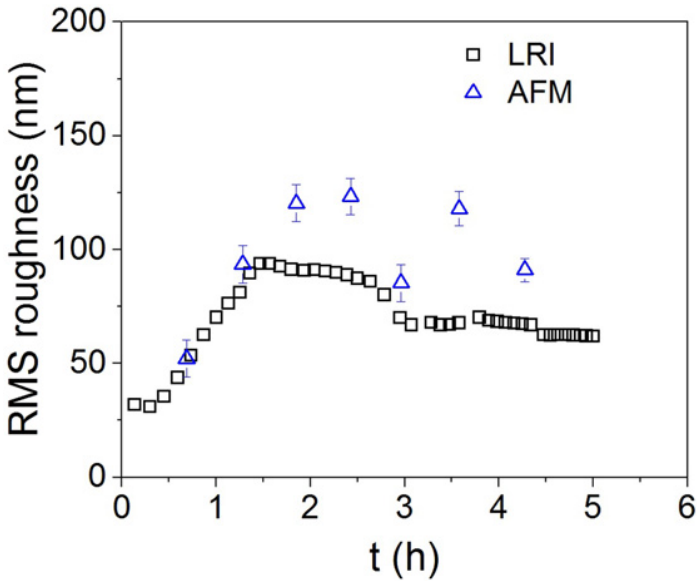

Fig. 5. Surface RMS roughness extracted from $R_{S c}$ (black squares) and measured with an AFM (blue triangles). The error bars refer to the experimental error of AFM roughness measurements.

process parameter such as the temperature or the gas composition but a parameter that is commonly used to correlate with the modification of crystal morphology according to the different growth rate of 100 and 111 faces. For instance, smooth surfaces can be obtained from a two steps growth involving first $\alpha \sim 2.8$ and then $\alpha<2.5[1,34,40]$.

Let's consider a crystal with only $\{111\}$ and $\{100\}$ faces and distant of $d$ from its nearest neighbor (as measured from respective crystals centers). It is possible to define the crystal density $\rho_{0}$ as a function of the mean nearest neighbor distance $\left(d_{0}\right)$ between crystals [41]:

$d_{0}=\frac{1}{2 \sqrt{\rho_{0}}}$

where $\rho_{0}$ refers to crystal density. This expression is calculated from a two dimensional space of randomly dispersed particles. It is derived from the probability that an arbitrary area of characteristic dimension $d_{0}$ contains only one particle. The angle $\gamma$ between two adjacent planes is $54.73^{\circ}$. According to Fig. 7 we note $x(\Delta t)$ the

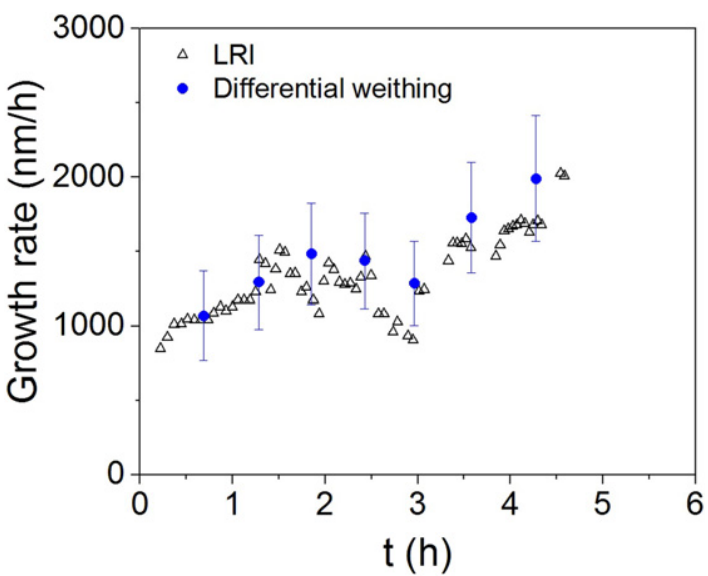

Fig. 6. Growth rate extracted from $R_{s p}$ (black triangles) and measured by differential weighting (blue circles).

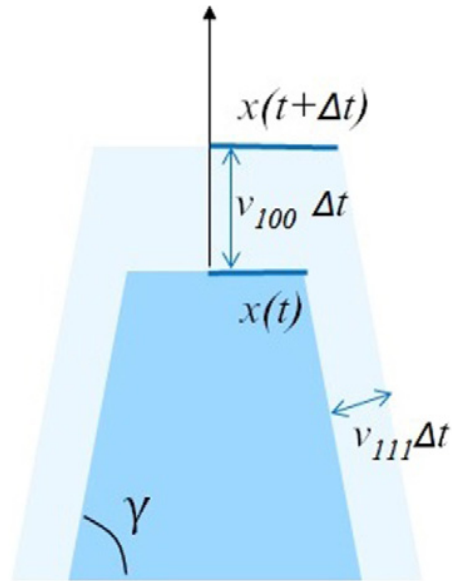

Fig. 7. Sketch of geometrical expansion of $\{111\}$ and $\{100\}$ edge.

expansion of the half length of $\{100\}$ plane for a time increase $\Delta t$. The following expression can be extracted geometrically[42]:

$x(\Delta t)=\left[\frac{v_{111}}{\sin (\gamma)}-v_{100} \cot (\gamma)\right] \Delta t$

Crystals are tilted so that the fastest growing direction is aligned with the substrate normal. The perpendicular to $\{100\}$ face is making an angle $\tau$ from the sample normal. Projection of length $x(\Delta t)$ on substrate plan $x^{\prime}(\Delta t)=x(\Delta t) \cos (\tau)$ with a direct relationship between $\cos (\tau)$ and growth parameter $\alpha$ as expressed by Ref. [8]:

$x^{\prime}(\Delta t)=\frac{x(\Delta t)}{\sqrt{1+(3 / \alpha-1)^{2}}}$

If growth proceeds with $2<\alpha<3$, and as long as a crystal does not reach its neighbor, faces grow freely and their surface scale up proportionally to $\Delta t^{2}$. When a crystal reaches a neighbor located

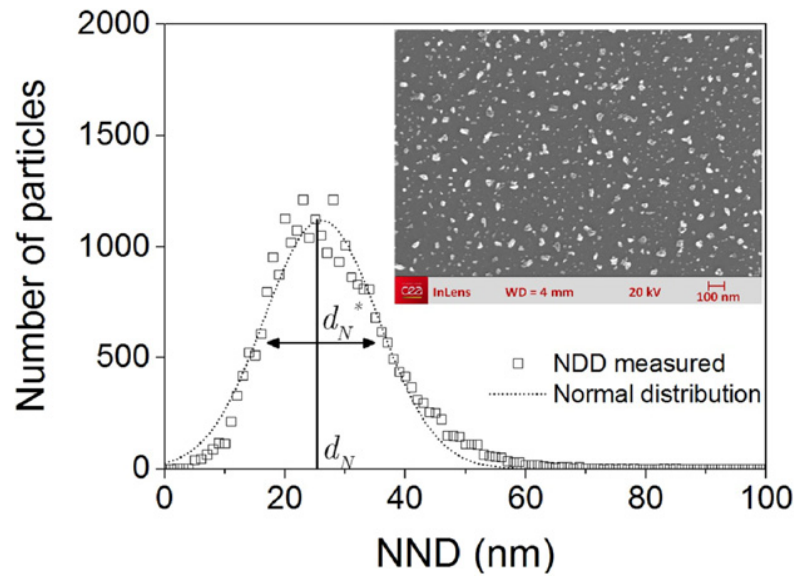

Fig. 8. Normal distribution of nearest neighbor distance (NND) denoted as $d_{N}$ in the text. It is measured via image treatment of the nanoseeding HRSEM picture (inset). 

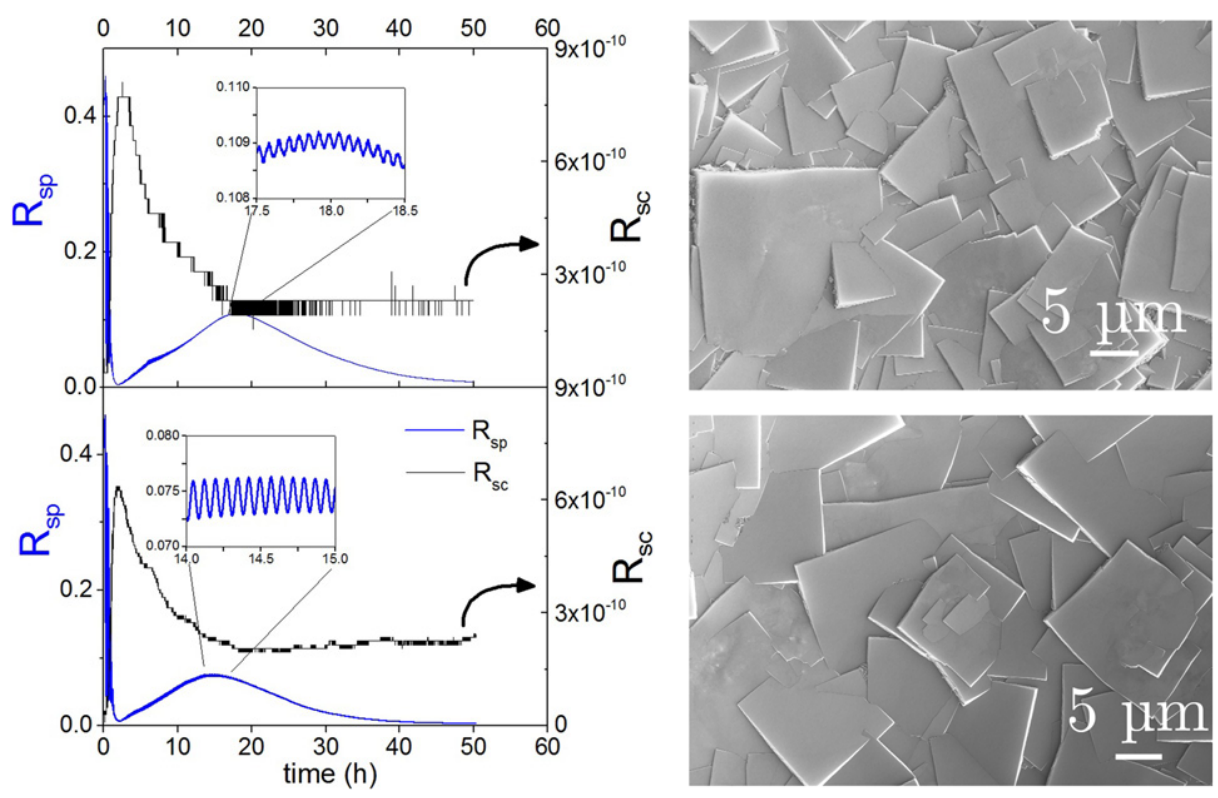

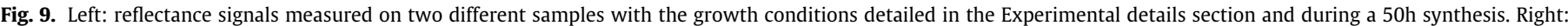
post-growth HRSEM pictures of the two films of about $100 \mu \mathrm{m}$ thickness.

at a distance $d$ we assume that if they have the same orientation, competition between crystals stops. Hence corresponding $\{100\}$ surface area does not vary anymore on the sample. This assumption is used in the following although a slight difference in the neighboring crystal orientations during a real growth eventually lead to some selection and a drop of the $\{100\}$ surface. Then, we consider the event of a crystal reaching a neighbor as a random variable and we can express the covering ratio $R_{100}$ as a function of time by the following statistic expression.

$R_{100}=\rho_{0}\left(x^{\prime}\right)^{2}(1-\mathcal{P})+\rho_{0} 4 d_{0}^{2} \mathcal{P}$

where $\mathcal{P}$ refers to the probability of a crystal to reach its nearest neighbor. Then we define $\rho_{N}$ as the initial nanoseeding density which is much higher than $\rho_{0}$ (and subsequent distance to nearest nanoseed $d_{N}$ ). The growth is here mainly initiated from the diamond nanoseeds. We introduce the selection rate $K$ as $\rho_{N} / \rho_{0}$ which refers to selection progress. In other words, only a fraction of the seeded nanodiamonds (of initial density $\rho_{N}$ ) emerge after the film coalescence and have a distinguishable $\{100\}$ face like the crystal on the sketch of Fig. 7. Their density is calculated as $\rho_{N} / K$. This statement can be understood by comparing the first (only seeded nanodiamonds) and third (oriented crystals) pictures of Fig. 2. Combining Eqs. (4) and (1), the covering ratio $R_{100}$ becomes:

$R_{100}=\rho_{0}\left(x^{\prime}\right)^{2}(1-\mathcal{P})+\mathcal{P}$

Now we assume that $d_{0}$ follows a normal distribution with mean value $d_{0}$ and standard deviation $d_{0}^{*}$ and the analytical expression of $\mathcal{P}$ depends on the error function:

$\mathcal{P}=\frac{1}{2}\left(1+\operatorname{erf}\left(\frac{x^{\prime} \sqrt{2}-d_{0}}{\sqrt{2} d_{0}^{*}}\right)\right)$

The choice of a normal distribution is based on the central limit theorem which consider that all the physical phenomenon involved in the adsorption of nanoseeds on the surface are random variables. Finally, the covering ratio $R_{100}$ is a function of five parameters: $\alpha, v_{100}, \rho_{N}, K$ and $d_{N}^{*}$. Before growth, a HRSEM picture of the nanoseeding gives access to $\rho_{N}$ (or $d_{N}$ ) and $d_{N}^{*}$, the nanoseeding distribution statistical parameters. Those parameters are measured through a simple algorithm of image treatment as presented on Fig. 8. They are related to the crystals statistical parameters by the multiplying factor $K$.

Hypotheses necessary to exploit this model are as following:

- Crystals verify $2<\alpha<3$ and have all the same orientation.

- Number of crystals involved in texture is much less than nanoseeding density $(K \gg 1)$.

- Nearest neighbor distance $\left(d_{0}\right)$ between crystals follows a normal distribution.

To correlate optical signal $R_{s c}$ and covering ratio $R_{100}$, the latter must be calculated during growth. As described earlier, parameters $\rho_{N}$ and $d_{N}^{*}$ are obtained before growth by HRSEM observation of the nanoseeded surface. Missing parameters $\left(K, v_{100}\right.$ and $\left.\alpha\right)$ can be obtained due to their influence on Expression (5). We can estimate their values by adjustment of the following growth rate expression:

$G=R_{100} v_{100}+\left(1-R_{100}\right) v_{111}$

where we assume growth rate as a weighted combination of each crystal orientation growth rate (individual weight being the corresponding orientation surface covering ratio).

\section{Discussion}

Potential correlation is investigated on two sets of data obtained from two identical growths performed with same conditions (see Experimental details) but at different times. Both reflectance signals are recorded during a fifty hours growth. While the step-bystep synthesis leads to overlapping issues between steps, the signals presented on Fig. 9 are easier to interpret.

Although the experience for two datasets have identical conditions, specular reflectance signal is slightly different because the 

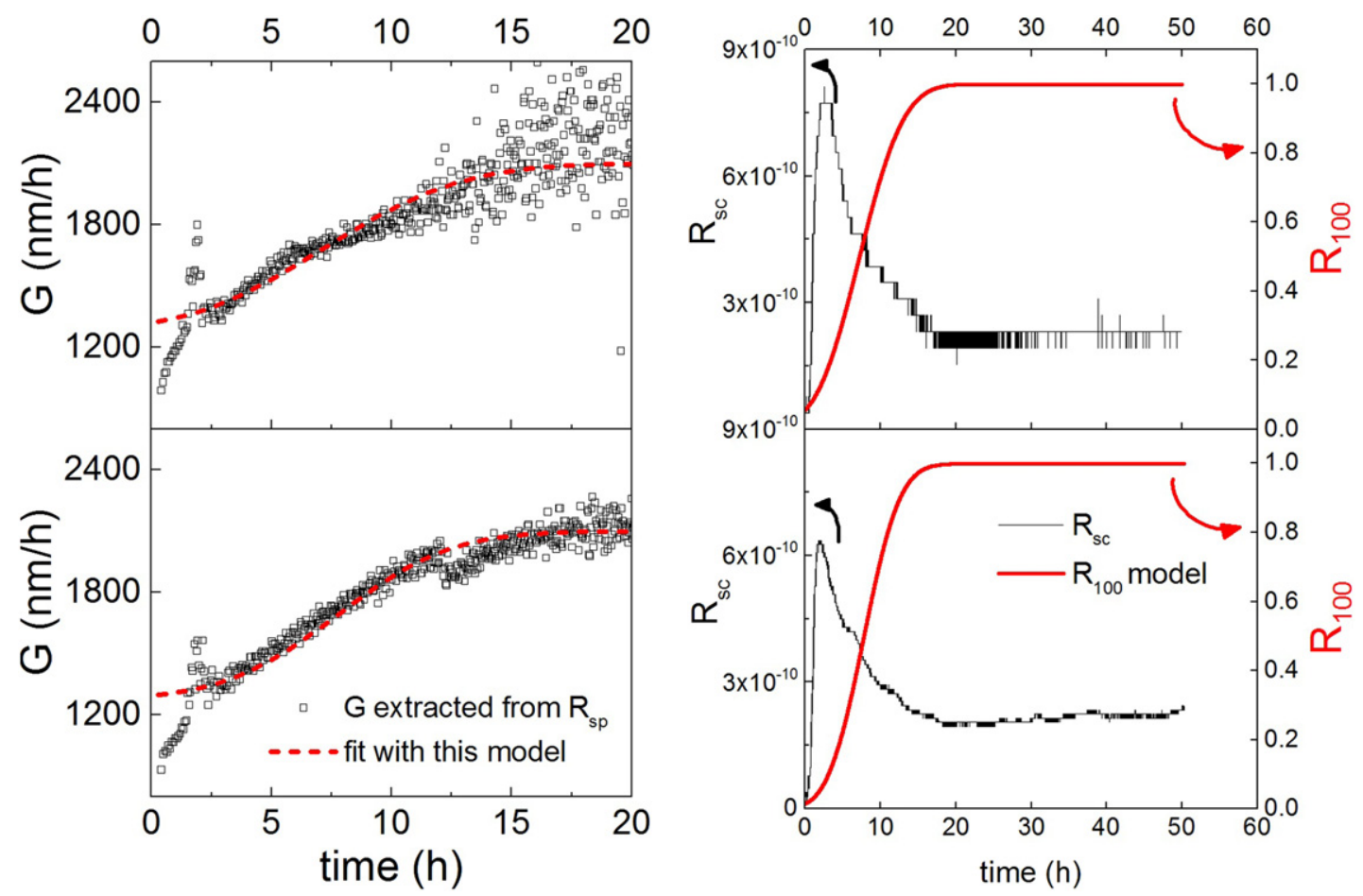

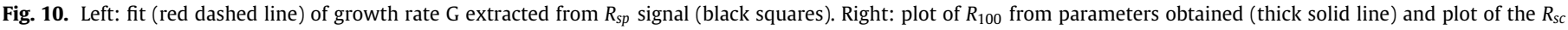
signal (thin dotted line)

laser probed zone is local and its position in the plasma can vary from one sample to another. Also could surface temperature be slightly different according to the probed position. Specular reflectance $R_{s p}$ presents interferences that cannot be seen on Fig. 9 (left) due to the large time scale but are presented in the insets. Amplitude is first decaying due to roughness increase and then increasing due to $\{100\}\{111\}\langle 100\rangle$ texture formation and consequent smoothening. After $20 \mathrm{~h}$, amplitude decays because of absorption in the film. Scattered reflectance $R_{S c}$ has opposite trends but is not affected by film absorption and remains relatively constant after $20 \mathrm{~h}$. This peculiarity makes it a perfect tool to follow morphology during long lasting growth. The morphology of the top surface of the film is presented on the right of Fig. 9. Films are about $100 \mu \mathrm{m}$ thick.

Specular reflectance of those two datasets are used to extract the growth rate evolution as presented on the left side of Fig. 10. As expected from the step-by-step synthesis, the growth rate is increasing as long as the slow growing faces are buried in the selection process. Statistical parameters of nanoseeding distribution are measured before the growth in both cases. Expression (7) is then used to fit experimental data and extract the remaining parameters for the model (as presented on Table 1). As the experimental conditions are similar, it is likely to have similar parameters for both growths. The growth parameter of these synthesis is consistent with the texture observed on HRSEM pictures of Fig. 9 and the formation of relatively smooth $\{100\}$ surface. A selection rate of about $10^{3}$ is slightly higher than the one calculated from Ref. [34]. But in our case it is an experimental value considered independent from $\alpha$ and $v_{100}$. For such film, the minimal thickness resulting in a jointed $\{100\}$ surface is between 15 and $20 \mu \mathrm{m}$ according to Ref. [34] which in our case correspond to covering ratio above $75 \%$.

From our fit, we extract values of $\alpha$ parameter in the range [2.83-2.86] which is compatible with the texture observed on both grown films in Fig. 9 (right). Furthermore, the growth rate is similar to what is observed on sequential growth and the $K$ parameters are higher than $10^{3}$, in agreement with our hypothesis. Thus extracted parameters values are compatible with previous observations, which is a first confirmation of the validity of our approach.

Once all six parameters are defined for each dataset, the covering ratio $R_{100}$ is calculated and compared to the scattered reflectance on Fig. 10 (right side). The surface of the film seems to achieve a stable covering ratio after $20 \mathrm{~h}$ right at the moment where $R_{s p}$ signal amplitude is maximum which means roughness is minimum. Stabilization of $R_{s c}$ occurs as neither roughness nor scattered light are evolving anymore. Stabilization of $R_{s c}$ can here be used as a sign of completeness of texture and show that our model developed is well describing texture formation according to considered hypothesis.
Table 1

List of the parameters obtained with this method for two identical synthesis. Statistical parameters of nanoseeding are the density $\rho_{N}$ and standard deviation of the nearest neighbor distance between nanoseeds $d_{N}^{*}$. Parameters of the synthesis are the selection rate $K$, the growth parameter $\alpha$ and the growth rate of $\{100\}$ faces.

\begin{tabular}{lll}
\hline Parameters & Dataset 1 & Dataset 2 \\
\hline$\rho_{N}\left(\mathrm{~cm}^{-2}\right)$ & $3.410^{10}$ & $4.210^{10}$ \\
$d_{N}^{*}(\mathrm{~nm})$ & 12.5 & 15.4 \\
$K$ & 1475 & 1078 \\
$\alpha$ & 2.83 & 2.86 \\
$v_{100}(\mathrm{~nm} / \mathrm{h})$ & 2100 & 2100 \\
\hline
\end{tabular}

\section{Conclusion}

A step-by-step growth of $\{100\}\{111\}\langle 100\rangle$ textured diamond film was performed. Specular reflectance of growing film can be correlated to ex situ characterizations in terms of roughness and growth rate. The formation of such a texture is believed to result in a significant growth rate increase. We also report a correlation between surface covering ratio $R_{100}$ and scattered light reflectance. This was interpreted after a thorough HRSEM study of the exact same area during a step-by-step synthesis where surface covering ratio $R_{100}$ of 
the surface is calculated and shows a similar behavior to the scattered reflectance signal recorded during growth. To further investigate this correlation, a simple analytical model was developed to calculate $R_{100}$ from several growth parameters such as $\alpha, \rho_{0}$. It was used on two synthesis made with different samples but with the same conditions to compare predicted $R_{100}$ and scattered reflectance. Our results indicate that this optical signal can be easily used to monitor texture evolution and the exact moment when $\{100\}$ faces join together to form a smooth surface.

\section{References}

[1] R.E. Clausing, L. Heatherly, L.L. Horton, E.D. Specht, G.M. Begun, Z.L. Wang, Texture and morphologies of chemical vapor deposited (CVD) diamond, Diam. Relat. Mater. 1 (5) (1992) 411-415.

[2] S. Barrat, I. Dieguez, H. Michel, E. Bauer-Grosse, Morphometric analysis of diamond crystals elaborated by microwave plasma assisted chemical vapour deposition: application to textured films, Diam. Relat. Mater. 3 (4) (1994) 520-524.

[3] S. Barrat, E. Bauer-grosse, Prediction of the feasibility of oriented diamond films by microwave plasma-assisted CVD, Diam. Relat. Mater. 4 (4) (1995) 419-424.

[4] A. Van der Drift, Evolutionary selection, a principle governing growth orientation in vapour-deposited layers, Philips Res. Rep. (1967)

[5] G.Z. Cao, J.J. Schermer, W.J.P. van Enckevort, W.A.L.M. Elst, L.J. Giling, Growth of $\{100\}$ textured diamond films by the addition of nitrogen, J. Appl. Phys. $79(3)$ (1996) 1357-1364.

[6] C. Wild, P. Koidl, W. Müller-Sebert, H. Walcher, R. Kohl, N. Herres, R. Locher, R. Samlenski, R. Brenn, Chemical vapour deposition and characterization of smooth $\{100\}$-faceted diamond films, Diam. Relat. Mater. 2 (2-4). (1993)

[7] C. Wild, N. Herres, P. Koidl, Texture formation in polycrystalline diamond films, J. Appl. Phys. 68 (3) (1990) 973-978.

[8] C. Wild, R. Kohl, N. Herres, W. Mller-Sebert, P. Koidl, Oriented CVD diamond films: twin formation, structure and morphology, Diam. Relat. Mater. 3 (4-6) (1994) 373-381.

[9] M. Rabarot, J. Widiez, S. Saada, J.P. Mazellier, C. Lecouvey, J.C. Roussin, J. Dechamp, P. Bergonzo, F. Andrieu, O. Faynot, S. Deleonibus, L. Clavelier, J.P. Roger, Silicon-on-diamond layer integration by wafer bonding technology, Diam. Relat. Mater. 19 (7-9) (2010) 796-805.

[10] C. Vivensang, L. Ferlazzo-Manin, M.F. Ravet, G. Turban, F. Rousseaux, A. Gicquel, Surface smoothing of diamond membranes by reactive ion etching process, Diam. Relat. Mater. 5 (6-8) (1996) 840-844.

[11] C. Blin, X. Checoury, H.A. Girard, C. Gesset, S. Saada, P. Boucaud, P. Bergonzo, Optical analysis of p-type surface conductivity in diamond with slotted photonic crystals, Adv. Opt. Mater. 1 (2013) 963-970.

[12] H. Fitouri, Z. Benzarti, I. Halidou, T. Boufaden, B.E. Jani, Modeling of laser reflectance evolution during metalorganic vapor phase epitaxy growth of GaN using SiN treatment, Appl. Surf. Sci. 253 (1) (2006) 258-260.

[13] E. Steinsland, T. Finstad, A. Hanneborg, Laser reflectance interferometry for in situ determination of silicon etch rate in various solution, J. Electrochem. Soc. 146 (10) (1999) 3890-3895.

[14] G.J. Glanner, H. Sitter, W. Faschinger, M.A. Herman, Evaluation of growth temperature, refractive index, and layer thickness of thin ZnTe, MnTe, and CdTe films by in situ visible laser interferometry, Appl. Phys. Lett. 65 (8) (1994) 998-1000.

[15] F. Chen, H. Lu, Z. Chen, T. Zhao, G. Yang, Optical real-time monitoring of the laser molecular-beam epitaxial growth of perovskite oxide thin films by an oblique-incidence reflectance-difference technique, J. Opt. Soc. Am. B 18 (7) (2001) 1031.

[16] J. Bajaj, S.J.C. Irvine, H.O. Sankur, S.A. Svoronos, Modeling of in situ monitored laser reflectance during MOCVD growth of HgCdTe, J. Electron. Mater. $22(8)$ (1993) 899-906.

[17] A.M. Bonnot, B.S. Mathis, S. Moulin, Investigation of the growth kinetics of low pressure diamond films by in situ elastic scattering of light and reflectivity, Appl. Phys. Lett. 63 (13) (1993) 1754-1756.
[18] A. Bonnot, B. Mathis, S. Moulin, Growth kinetic analysis of diamond films by in-situ elastic scattering of light and reflectivity, Diam. Relat. Mater. 3 (4-6) (1994) 426-430.

[19] S.A. Catledge, P. Baker, J.T. Tarvin, Y.K. Vohra, Multilayer nanocrystalline/ microcrystalline diamond films studied by laser reflectance interferometry, Diam. Relat. Mater. 9 (8) (2000) 1512-1517.

[20] M. Kraszewski, R. Bogdanowicz, Laser reflectance interferometry system with a $405 \mathrm{~nm}$ laser diode for in situ measurements of CVD diamond thickness, Met Meas. Syst. 20 (4).(2013)

[21] W.G. Breiland, K.P. Killeen, A virtual interface method for extracting growth rates and high temperature optical constants from thin semiconductor films using insitu normal incidence reflectance, J. Appl. Phys. 78 (11) (1995) 6726-6736.

[22] H.A. Girard, S. Perruchas, C. Gesset, M. Chaigneau, L. Vieille, J.-C. Arnault, P. Bergonzo, J.-P. Boilot, T. Gacoin, Electrostatic grafting of diamond nanoparticles: a versatile route to nanocrystalline diamond thin films, ACS Appl. Mater. Interfaces 1 (12) (2009) 2738-2746.

[23] C.A. Schneider, W.S. Rasband, K.W. Eliceiri, NIH Image to Imagel: 25 years of image analysis, Nat. Methods 9 (7) (2012) 671-675.

[24] S.K. Nayar, K. Ikeuchi, T. Kanade, Surface reflection: physical and geometrical perspectives, IEEE Trans. Pattern Anal. Mach. Intell. 13 (7) (1991) 611-634.

[25] C.D. Zuiker, D.M. Gruen, A.R. Krauss, In situ laser reflectance interferometry measurement of diamond film growth, J. Appl. Phys. 79 (7) (1996) 3541-3547.

[26] S. Barrat, P. Pigeat, E. Bauer-Grosse, Three-dimensional simulation of CVD diamond film growth, Diam. Relat. Mater. 5 (3-5) (1996) 276-280.

[27] E. Hecht, Optics, second ed., Addison-Wesley, Reading, Mass, 1987.

[28] S. Dunst, H. Sternschulte, M. Schreck, Growth rate enhancement by nitrogen in diamond chemical vapor deposion, a catalytic effect, Appl. Phys. Lett. 94 (22) (2009) 224101.

[29] C.-H. Wu, W.H. Weber, T.J. Potter, M.A. Tamor, Laser reflective interferometry for in situ monitoring of diamond film growth by chemical vapor deposition, J. Appl. Phys. 73 (6) (1993) 2977-2982.

[30] F de Theije, J. Schermer, W. van Enckevort, Effects of nitrogen impurities on the CVD growth of diamond: step bunching in theory and experiment, Diam. Relat. Mater. 9 (8) (2000) 1439-1449.

[31] J. Achard, F. Silva, O. Brinza, A. Tallaire, A. Gicquel, Coupled effect of nitrogen addition and surface temperature on the morphology and the kinetics of thick CVD diamond single crystals, Diam. Relat. Mater. 16 (4) (2007) 685-689.

[32] C.-S. Yan, Y.K. Vohra, Multiple twinning and nitrogen defect center in chemical vapor deposited homoepitaxial diamond, Diam. Relat. Mater. 8 (11) (1999) 2022-2031.

[33] W. Müller-Sebert, E. Wörner, F. Fuchs, C. Wild, P. Koidl, Nitrogen induced increase of growth rate in chemical vapor deposition of diamond, Appl. Phys. Lett. 68 (6) (1996) 759.

[34] C. Nørgård, A. Matthews, Two-step diamond growth for reduced surface roughness, Diam. Relat. Mater. 5 (3-5) (1996) 332-337.

[35] P.W. May, N.L. Allan, M.N.R. Ashfold, J.C. Richley, Y.A. Mankelevich, Simulations of polycrystalline CVD diamond film growth using a simplified Monte Carlo model, Diam. Relat. Mater. 19 (5-6) (2010) 389-396.

[36] C. Ophus, E. Luber, D. Mitlin, Simulations of faceted polycrystalline thin films: asymptotic analysis, Acta Mater. 57 (5) (2009) 1327-1336.

[37] A.B. Rodriguez-Navarro, Model of texture development in polycrystalline films growing on amorphous substrates with different topographies, Thin Solid Films $389(1-2)(2001) 288-295$.

[38] X. Jiang, G. Yu, S.T. Lee, Simulation of polycrystalline 3D film growth: an investigation of the evolution of grain size and texture in diamond films, Appl. Phys. A 74 (2) (2002) 217-224

[39] A. Bogatskiy, J.E. Butler, A geometric model of growth for cubic crystals: diamond, Diam. Relat. Mater. 53 (2015) 58-65.

[40] C. Nørgård, S.S. Eskildsen, A. Matthews, The influence of oriented growth on the surface roughness of CVD diamond films, Surf. Coat. Technol. 74-75 (Part 1) (1995) 358-361.

[41] J.P. Clark, F.C. Evans, Distance to nearest neighbor as measure of spatial relationship in populations, Ecol. 35 (4) (1954) 445-453.

[42] Y. von Kaenel, J. Stiegler, E. Blank, O. Chauvet, C. Hellwig, K. Plamann, Microstructure evolution and defect incorporation in highly oriented and textured CVD diamond films, Phys. Status Solidi (a) 154 (1) (1996) 219-238. 